\title{
Exclusive diffractive photon bremsstrahlung at high energies
}

Piotr LEBIEDOWICZ*

Institute of Nuclear Physics PAN, PL-31-342 Cracow, Poland

E-mail: Piotr.Lebiedowicz@ifj.edu.pl

\section{Antoni SZCZUREK ${ }^{\dagger}$}

University of Rzeszów, PL-35-959 Rzeszów, Poland, and

Institute of Nuclear Physics PAN, PL-31-342 Cracow, Poland

E-mail: Antoni.Szczurek@ifj.edu.pl

\begin{abstract}
We discuss the $p p \rightarrow p p \gamma$ reaction at the LHC. We consider diffractive classical bremsstrahlung mechanisms including effects of non point-like nature of protons. In addition, we take into account (vector meson)-pomeron, photon-pion as well as photon-pomeron exchange processes for the first time in the literature. The integrated diffractive bremsstrahlung cross section $\left(E_{\gamma}>100 \mathrm{GeV}\right)$ is only of the order of $\mu \mathrm{b}$. We try to identify regions of the phase space where one of the mechanisms dominates. The classical bremsstrahlung dominates at large forward/backward photon pseudorapidities, close to the pseudorapidities of scattered protons. In contrast, the photon-pomeron (pomeron-photon) mechanism dominates at midrapidities but the related cross section is rather small. In comparison the virtual-omega-rescattering mechanism contributes at smaller angles of photons (larger photon rapidities).
\end{abstract}

XXI International Workshop on Deep-Inelastic Scattering and Related Subject-DIS2013,

*This work was supported by the Polish National Science Centre (on the basis of decision No. DEC2011/01/N/ST2/04116 and DEC-2011/01/B/ST2/04535).

${ }^{\dagger}$ Speaker. 


\section{Introduction}

Exclusive diffractive photon bremsstrahlung mechanism have been studied recently [1] in the $p p \rightarrow p p \gamma$ reaction at the LHC. ${ }^{1}$ Because at high energy the pomeron exchange is the driving mechanism of bremsstrahlung it is logical to call the mechanisms described by the diagrams from (a) to (d) in Fig.1 the diffractive bremsstrahlung to distinguish from the low-energy bremsstrahlung driven by meson exchanges.

We shall include bremsstrahlung diagrams as well as some new diagrams characteristic exclusively for proton-proton scattering, not present e.g. in $e^{+} e^{-}$scattering. We include diagrams which arise in the vector-dominance model as well as photon-pion (pion-photon) and photon-pomeron (pomeron-photon) exchange processes not discussed so far in the literature. We shall try to identify the region of the phase space where one can expect a dominance of one of the processes through detailed studies of several differential distributions. The exclusive photon production mechanism is similar to $p p \rightarrow p p \omega$ [2] and $p p \rightarrow p p \pi^{0}$ [3] processes. As discussed in the past the dominant hadronic bremsstrahlung-type mechanism is the Drell-Hiida-Deck mechanism [4] for diffractive production of $\pi N$ final states (for a nice review we refer to [5] and references therein). The photons radiated off the initial and final state protons can be seen by the Zero Degree Calorimeters (ZDCs) [7] in the pseudorapidity region $|\eta|>8.5$ and 8.3 at the CMS and ATLAS, respectively. ${ }^{2}$ The scattered protons can be tagged e.g. by the ALFA detectors at ATLAS (see [9]) or TOTEM at CMS.

\section{Sketch of formalism}

The amplitudes for exclusive production of photons shown schematically by diagrams in Fig.1 are discussed elsewhere [1], e.g. formula of diagram (a) can be written as

$$
\begin{aligned}
\mathscr{M}_{\lambda_{a} \lambda_{b} \rightarrow \lambda_{1} \lambda_{2} \lambda_{3}}^{(a)}= & e \bar{u}\left(p_{1}, \lambda_{1}\right) \phi^{*}\left(p_{3}, \lambda_{3}\right) S_{N}\left(p_{1 f}^{2}\right) \gamma^{\mu} u\left(p_{a}, \lambda_{a}\right) F_{\gamma N^{*} N}\left(p_{1 f}^{2}\right) F_{I P N N^{*}}\left(p_{1 f}^{2}\right) \\
& \times i s C_{\mathbb{P}}^{N N}\left(\frac{s}{s_{0}}\right)^{\alpha_{\mathbb{P}}\left(t_{2}\right)-1} \exp \left(\frac{B_{\mathbb{P}}^{N N} t_{2}}{2}\right) \frac{1}{2 s} \bar{u}\left(p_{2}, \lambda_{2}\right) \gamma_{\mu} u\left(p_{b}, \lambda_{b}\right) .
\end{aligned}
$$

We use interaction parameters of Donnachie-Landshoff [10] fixed to the total and elastic $\pi N$ and $N N$ scattering, see [11]. In the bremsstrahlung processes discussed here the intermediate nucleons are off-mass shell. In our approach the off-shell effects related to the non-point-like protons in the intermediate state are included by the following simple extra form factors

$$
F\left(p^{2}\right)=\frac{\Lambda_{N}^{4}}{\left(p^{2}-m_{p}^{2}\right)^{2}+\Lambda_{N}^{4}} .
$$

The cut-off parameters $\Lambda_{N}$ in the form factors are not known but could be fitted in the future to the (normalized) experimental data. We shall discuss how the uncertainties of the form factors influence our final results.

\footnotetext{
${ }^{1}$ The $p p \rightarrow p p \gamma$ process at high energies was discussed before in [6] and it was proposed to use the exclusive photon bremsstrahlung to measure or estimate elastic proton-proton cross section at the LHC. But only the approximate formulas for the classical bremsstrahlung were given there. The participating particles were treated there as point-like particles. No differential distributions for the exclusive bremsstrahlung have been discussed.

${ }^{2}$ The exclusive $p p \rightarrow n n \pi^{+} \pi^{+}$[8] can be also measured with the help of the ZDCs. Very large cross sections has been found which is partially due to interference of a few mechanisms.
} 
(a)
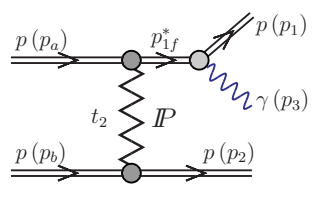

(e)

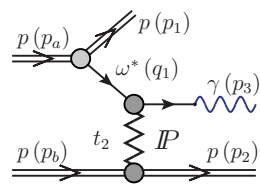

(i)

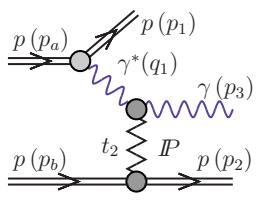

(b)
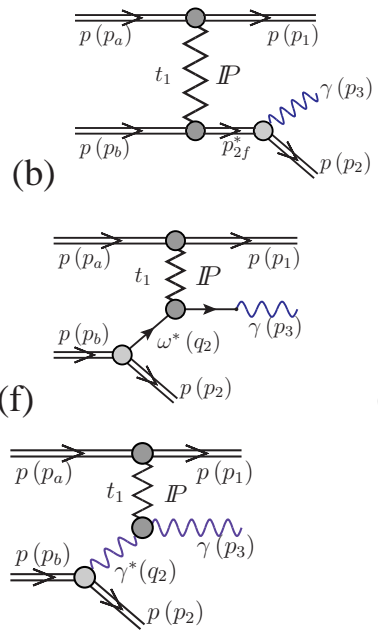

(c)
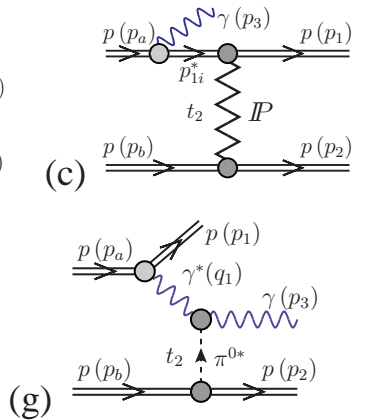

(d)

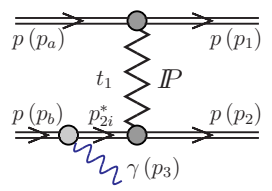

(h)

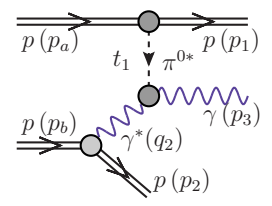

Figure 1: Diagrams of the dominant diffractive bremsstrahlung (a) - (d) driven by the pomeron exchange, and from (e) to (j), the virtual $\omega$ - pomeron exchanges (or $\omega$-rescattering), the $\gamma-\pi^{0}$ exchanges, and the $\gamma$ pomeron exchanges (or $\gamma$-rescattering).

\section{Results}

In the following section we show results of the differential distributions for the all mechanisms of Fig.1. In calculating cross section we perform integrations in $\xi_{1}=\log _{10}\left(p_{1 \perp} / 1 \mathrm{GeV}\right)$ and $\xi_{2}=\log _{10}\left(p_{2 \perp} / 1 \mathrm{GeV}\right)$ instead in $p_{1 \perp}$ and $p_{2 \perp}$, in the photon pseudorapidity $\eta_{\gamma}$ and the relative azimuthal angle between the outgoing protons $\phi_{12}=\phi_{1}-\phi_{2}$.

Corresponding distributions in the photon energy and the transverse momentum are shown in Fig.2. The ZDC detectors can measure only photons above some energy threshold. In the calculation of classical bremsstrahlung presented here we assume $E_{\gamma}>100 \mathrm{GeV}$ as an example. The contribution of classical bremsstrahlung is concentrated at very small transverse momenta which is consistent with very small photon emission angle (large pseudorapidity). The other distributions have rather similar shape and vanish at $p_{\perp, \gamma}=0 \mathrm{GeV}$. The exact shape may depend somewhat on the functional form and values of cut-off parameters of off-shell form factors taking into account the non-point-like nature of the vertices involved. Here we have fixed the values of the corresponding form factors at typical hadronic scales.

In Fig. 3 we show two-dimensional distributions for the classical bremsstrahlung in $\left(\xi_{1}, \xi_{2}\right)$ in a full range of photon (pseudo)rapidity and in $\left(t_{1}, t_{2}\right)$ for $\eta_{\gamma}>0$. We observe an enhancement along the diagonal. This enhancement is a reminiscence of the elastic scattering for which $\xi_{1}=\xi_{2}$ or $t_{1}=t_{2}$. The distributions discussed here could in principle be obtained with the TOTEM detector at CMS to supplement the ZDC detector for the measurement of photons.

Photon (pseudo)rapidity distribution is particularly interesting. In Fig.4 we show both distributions for photons $\eta_{\gamma}$ (left panel) and corresponding distributions for outgoing protons $\eta_{p}$ (right panel). In this variable both protons and photons are localized in a similar region of pseudorapidities (or equivalently polar angles). The classical bremsstrahlung clearly gives the largest contribution. It is also concentrated at very large $\eta_{\gamma}$ i.e. in the region where ZDC detectors can be used. We observe a large cancellation between the corresponding terms in the amplitude (as shown in 

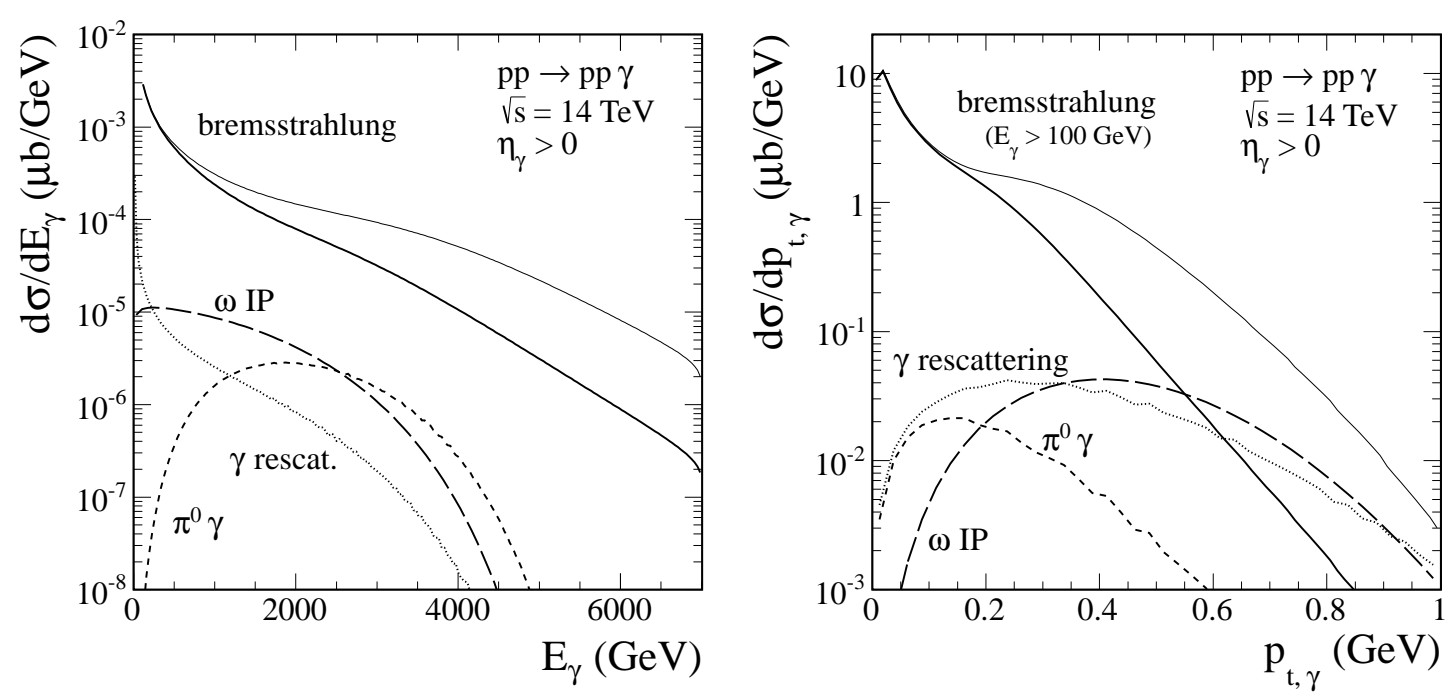

Figure 2: Energy spectrum of photons (left panel) and distribution in transverse momentum of photons (right panel) for all processes considered here at $\sqrt{s}=14 \mathrm{TeV}$ and for $\eta_{\gamma}>0$. For classical bremsstrahlung we have imposed $E_{\gamma}>100 \mathrm{GeV}$ and used two values of $\Lambda_{N}=0.8,1 \mathrm{GeV}$ in the proton off-shell form factors (see the lower and upper solid line, respectively).
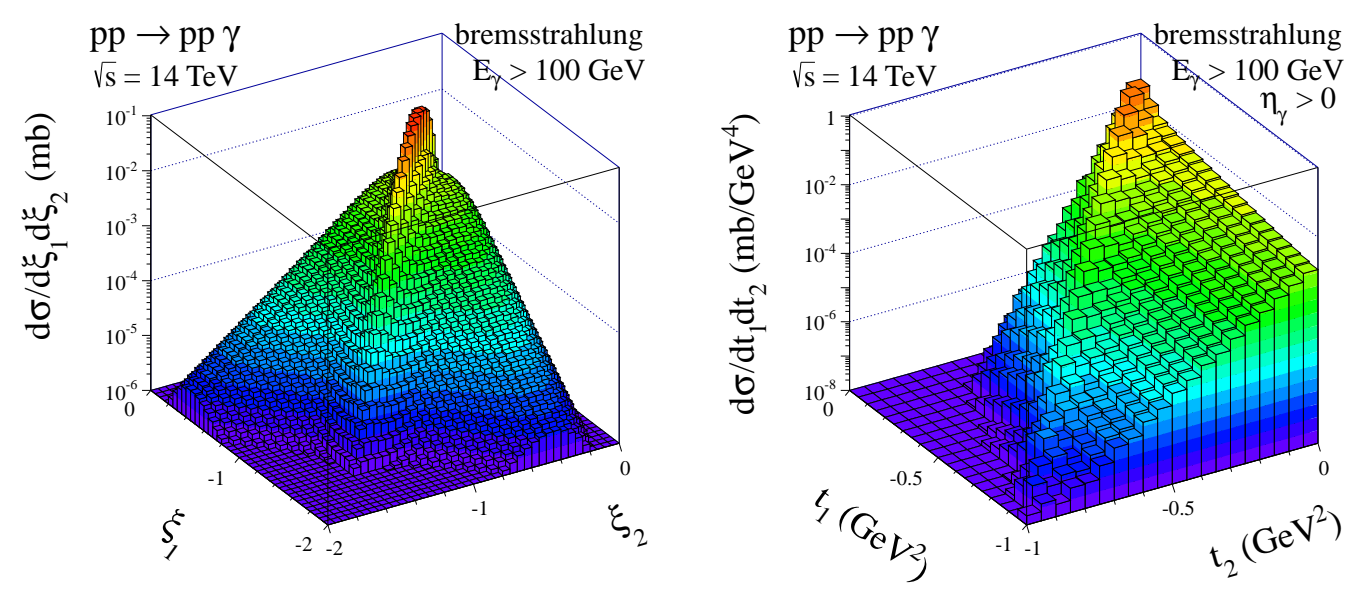

Figure 3: Distribution in $\left(\xi_{1}, \xi_{2}\right)=\left(\log _{10}\left(p_{1 \perp} / 1 \mathrm{GeV}\right), \log _{10}\left(p_{2 \perp} / 1 \mathrm{GeV}\right)\right)\left(\right.$ left panel) and in $\left(t_{1}, t_{2}\right)$ (right panel) for the classical bremsstrahlung mechanisms at $\sqrt{s}=14 \mathrm{TeV}$. We have imposed in addition $E_{\gamma}>$ $100 \mathrm{GeV}$ and used $\Lambda_{N}=1 \mathrm{GeV}$.

the left panel between diagrams (a) and (c) denoted by the blue long-dashed and the blue shortdashed lines, respectively). The $\gamma$-rescattering process with pomeron exchange clearly dominates in the region of $\eta_{\gamma}<6$. The cross section for this process is rather small. Clearly an experimental measurement there would be a challenge.

If both protons are measured one could also study correlations in the relative azimuthal angle between outgoing protons. Our model calculations are shown in Fig.5. One can observe a large enhancement at back-to-back configurations for the classical bremsstrahlung which reminds the 

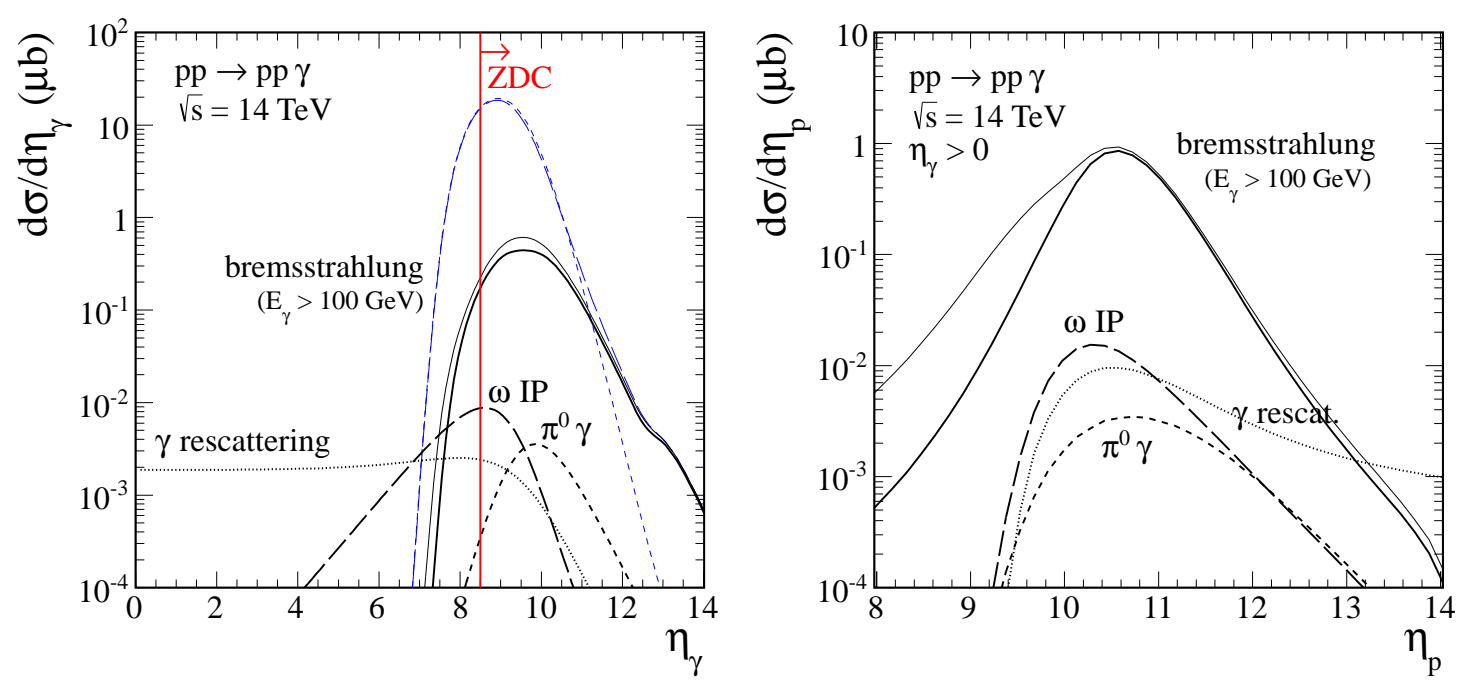

Figure 4: Distribution in (pseudo)rapidity of emitted photons (left panel) and in pseudorapidity of outgoing protons (right panel) at $\sqrt{s}=14 \mathrm{TeV}$. For classical bremsstrahlung we have imposed $E_{\gamma}>100 \mathrm{GeV}$ and used two values of $\Lambda_{N}=0.8,1 \mathrm{GeV}$ (the lower and upper solid line, respectively). The lower pseudorapidity limit for the CMS ZDC detector $\left(\eta_{\gamma}>8.5\right)$ is shown in addition by the vertical line.

elastic scattering case $\left(\phi_{12}=\pi\right)$. The contributions of other mechanisms are significantly smaller and weakly depend on $\phi_{12}$.

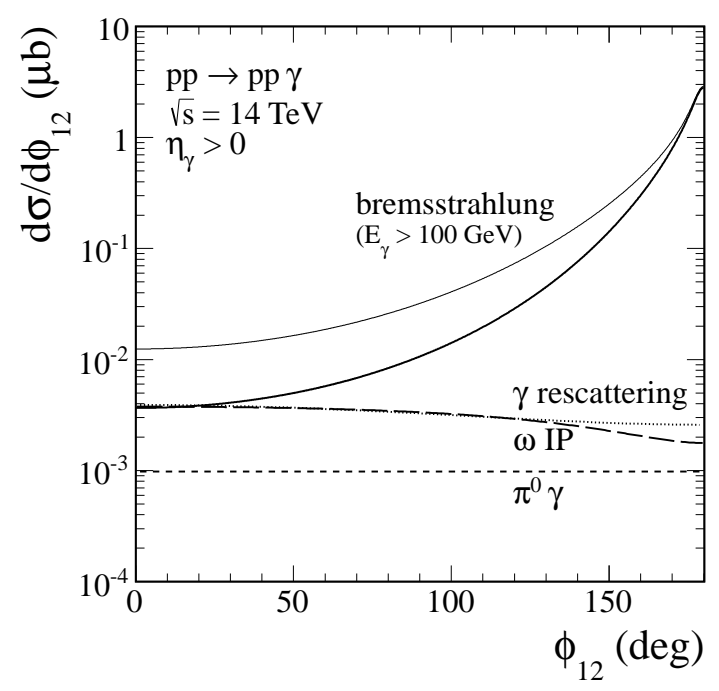

Figure 5: Distribution in relative azimuthal angle between outgoing protons for different mechanisms at $\sqrt{s}=14 \mathrm{TeV}$ and for $\eta_{\gamma}>0$. For classical bremsstrahlung we have imposed $E_{\gamma}>100 \mathrm{GeV}$ and used two values of $\Lambda_{N}=0.8,1 \mathrm{GeV}$ (the lower and upper solid line, respectively).

\section{Conclusions}

We have considered several mechanisms of exclusive single photon production and calculated 
differential distributions at the LHC nominal energy. By imposing several cuts one could select or enhance the contribution of one of the mechanisms. The classical bremsstrahlung mechanism turned out to give the biggest cross section concentrated at large photon (pseudo)rapidities. The photons are emitted at only slightly smaller pseudorapidities than the scattered protons. We observe a strong cancellation between the initial and final state radiation. The cross section for the classical bremsstrahlung is peaked at back-to-back configurations (similar transverse momenta or polar angles of outgoing protons and relative azimuthal angle concentrated close to $\phi_{12}=\pi$ ). This is a clear reminiscence of elastic scattering. Cut on photon energy $\left(E_{\gamma}>100 \mathrm{GeV}\right)$ reduces the region of $\phi_{12} \cong \pi$ significantly and the integrated diffractive bremsstrahlung cross section is only of the order of $\mu \mathrm{b}$. The cross section for pion-photon or photon-pion exchanges is much smaller. Here both small (photon exchange) and large (pion exchange) four-momentum transfers squared are possible. For this process there is no correlation in azimuthal angle between outgoing protons. The classical bremsstrahlung mechanisms could be studied in a close future with the help of Zero Degree Calorimeters (photons) and the ALFA or TOTEM detectors (protons).

Summarizing, even present LHC equipment allows to study exclusive production of photons. Since this process was never studied at high energies it is worth to make efforts to obtain first experimental cross sections. Since the cross sections are reasonably large one could try to obtain even some differential distributions. This would allow to test our understanding of the diffractive processes and help in pinning down some hadronic and electromagnetic off-shell form factors, difficult to test otherwise.

\section{References}

[1] P. Lebiedowicz and A. Szczurek, in print of Phys. Rev. D, [hep-ph/1303.2882].

[2] A. Cisek, P. Lebiedowicz, W. Schäfer and A. Szczurek, Phys. Rev. D83 (2011) 114004.

[3] P. Lebiedowicz and A. Szczurek, Phys. Rev. D87 (2013) 074037; P. Lebiedowicz and A. Szczurek, Diffractive mechanisms in $p p \rightarrow p p \pi^{0}$ reaction at high energies, in proceedings of DIS2013 conference.

[4] S.D. Drell and K. Hiida, Phys. Rev. Lett. 7 (1961) 199; R.T. Deck, Phys. Rev. Lett. 13 (1964) 169.

[5] A. B. Kaidalov, Phys. Rept. 50 (1979) 157; G. Alberi and G. Goggi, Phys. Rep. 74 (1981) 1; K.A. Goulianos, Phys. Rept. 101 (1983) 169.

[6] V.A. Khoze, J.W. Lämsä, R. Orava and M.G. Ryskin, JINST 6 (2011) P01005; H. Grönqvist, V.A. Khoze, J.W. Lämsä, M. Murray and R. Orava, [hep-ex/1011.6141].

[7] O.A. Grachov et al. (CMS Collaboration), J. Phys. Conf. Ser. 160 (2009) 012059; P. Jenni, M. Nessi and M. Nordberg, Report Nos. LHCC-I-016, CERN-LHCC-2007-001.

[8] P. Lebiedowicz and A. Szczurek, Phys. Rev. D83 (2011) 076002.

[9] R. Staszewski, P. Lebiedowicz, M. Trzebiński, J. Chwastowski and A. Szczurek, Acta Phys. Polon. B42 (2011) 1861.

[10] A. Donnachie and P.V. Landshoff, Phys. Lett. B296 (1992) 227.

[11] P. Lebiedowicz and A. Szczurek, Phys. Rev. D81 (2010) 036003. 